\title{
Communication Models for Throughput Optimization in Mesh Networks
}

\author{
Peng Wang Stephan Bohacek \\ Department of Electrical and Computer Engineering \\ University of Delaware \\ Newark, DE, USA \\ pwangee@udel.edu, bohacek@udel.edu
}

\begin{abstract}
There has been extensive research focused on maximizing the throughput of wireless networks in general and mesh networks in particular. Recently, techniques have been developed that reliably compute the maximum throughput of mesh networks. However, different approaches to throughput optimization make different assumptions on the physical layer's abilities. These assumptions are encapsulated in the communication model. This paper defines and compares a number of communication models, and proposes a general SINR Protocol Model which can apply the graph-based computation techniques and quite accurately represent the interference. Even ignoring the multi-conflicts, the performance of computed schedule from SINR Protocol Model is close to the theoretical throughput when applied to a realistic physical layer. Moreover, when techniques to correct the multi-conflicts are used, the actual performance is no worse than the theoretical performance.
\end{abstract}

\section{Categories and Subject Descriptors}

C.2.1 [Computer-Communication Networks]: Network Architecture and Design-Wireless Communication

\section{General Terms}

Performance

\section{Keywords}

Communication Model; Capacity Optimization; Mesh Network

\footnotetext{
${ }^{*}$ This work was prepared through collaborative participation in the Collaborative Technology Alliance for Communications and Networks sponsored by the U.S. Army Research Laboratory under Cooperative Agreement DAAD1901-2-0011. The U.S. Government is authorized to reproduce and distribute reprints for Government purposes notwithstanding any copyright notation thereon.
}

Permission to make digital or hard copies of all or part of this work for personal or classroom use is granted without fee provided that copies are not made or distributed for profit or commercial advantage and that copies bear this notice and the full citation on the first page. To copy otherwise, to republish, to post on servers or to redistribute to lists, requires prior specific permission and/or a fee.

PE-WASUN'08, October 27-28, 2008, Vancouver, BC, Canada.

Copyright 2008 ACM 978-1-60558-236-8/08/10 ...\$5.00.

\section{INTRODUCTION}

By providing connectivity to mobile users, mesh networks are poised to become a major extension of the Internet. More than 300 cities and towns have plans to deploy mesh networks, and several dozen cities have already deployed mesh networks. Some deployments have been in larger cities such as Corpus Christi's 147 sq. mile deployment. These mesh networks are meant to enhance city and emergency services communication as well as to provide city-wide, lowcost, ubiquitous Internet access for residents and visitors. Such networks promise to bring dramatic changes to data accessibility and hence have a major impact on society.

Coverage and throughput are two critical problems facing urban mesh networks. The throughput of wireless networks has been the focus of extensive research (e.g., [6, 7, 3, 4]). However, significant progress has been made in computing the throughput of urban mesh networks. Specifically, in [2], a computational strategy was developed that has been found to either compute the exact throughput or provide an estimate that is within $5 \%$ of the actual throughput. With these computational techniques, it is now possible to investigate the optimal throughput of realistic urban mesh networks.

The research efforts focused on throughput maximization model the communication in a wide variety of ways. These techniques are widely divided into two classes, namely protocol communication models and physical communication models. In the physical models, the data rate depends on the $S I N R$, and the interference can be from multiple sources. One drawback of such models is that they do not easily lend themselves to representations as a graph. The protocol models are an alternative class of models that does allow graph-based analysis of communication and throughput algorithms. For this reason, the protocol model is widely used. However, the traditional protocol models, such as Node Exclusive, 2-hop Node Exclusive and Sensing, have the drawback that they do not accurately model interference and can not deal with the interference from multiple sources. Thus, a general SINR protocol model is proposed to more accurately represent the interference.

Although a high theoretical throughput is provided, it is found that the traditional protocol models, such as Node Exclusive, 2-hop Node Exclusive and Sensing, result in very low actual throughput when applied to a realistic physical model. However, even interference from multiple sources is neglected, the SINR protocol model exhibits good throughput when applied to a physical model. If the techniques to correct multi-conflicts, including adding multi-conflicts constraints and adjusting the link bit rate, are applied, then the final scheduling is feasible and the actual throughput is no worse than the theoretical one. 
Furthermore, if we adjust the link bit rate for the optimal schedule found from SINR model, it is not necessary to fix the multi-conflicts by adding multi-conflicts constraints. In other word, if we adjust the link bit rate for the optimal schedule, we can ignore the multi-conflicts when we compute the optimal schedule. The multi-conflicts in the optimal schedule are removed by just adjusting the link bit rate, and the actual performance no worse than the theoretical one and in some cases, can be considerably higher.

The paper is organized as follows. The precise definitions of the models are provided in Section 2. Then, the techniques to compute the optimal schedule for protocol models are explored in Section 3. Moreover, techniques to correct interference from multiple sources are developed for SINR model in Section 3.4. Finally, the performance of the protocol models is examined in Section 4.

\section{COMMUNICATION MODELS}

The throughput of a network depends on the capabilities of the MAC and physical layer. However, including a detailed model of communication can complicate the development and analysis of computation schemes. Hence, simplifications are often made. The communication models found in the literature can be divided into two classes, namely protocol communication models and physical communication models.

\subsection{Physical Communication Models}

\subsubsection{Without ACKs}

Today's physical layers, such as $802.11 \mathrm{a} / \mathrm{b} / \mathrm{g}$ support a set of modulation and coding schemes. Each scheme coincides with a particular relationship between SNR and bit-error probability where the impacts of delay spread and Doppler spread are ignored. We assume that $M$ modulation schemes provide bit-rates $B R(1), B R(2), \cdots, B R(M)$. The probability of successful packet transmission with the $m$ th modulation scheme is denoted $P S P_{Z}(m, S I N R)$, where the subscript $Z$ denotes the packet size.

Hence, if links $a, b, \cdots, c$ are transmitting, then the probability of successful transmission of a packet of length $Z$ across link $x$ with modulation scheme $m$ is denoted $P S P_{Z}$ $\left(m, S I N R_{a, b, \ldots c,}^{t, r}(x)\right)$, where

$$
S I N R_{a, b, \ldots, c}^{t, r}(x):=\frac{H_{x, x}^{t, r}}{H_{a, x}^{t, r}+H_{b, x}^{t, r}+\cdots+H_{c, x}^{t, r}+N O I S E},
$$

where NOISE is the noise power and $H_{x, x}^{t, r}$ is the normalized channel gain across link $x$. That is $H_{x, x}^{t, r}$ is the signal strength at the receiver of link $x$ due to the data transmission by the transmitter of link $x$. Similarly, $H_{a, x}^{t, r}$ is the signal strength at the receiver of link $x$ due to data transmissions by the transmitter of link $a$. Note that the transmission power is embedded into $H_{a, x}^{t, r}$. Thus, if the channel gain from the transmitter of link $a$ to the receiver of link $x$ is $h_{a, x}$, and link $a$ transmits with power $p_{a}$, then $H_{a, x}^{t, r}=h_{a, x} p_{a}$. If a link can transmit at different powers, we represent that link are multiple distinct links between the same transmitter and receiver. By convention, $H_{x, y}^{t, r}=\infty$ if $x$ 's transmitter is $y$ 's receiver. Hence, in this case, if data is transmitted across link $x$, then it is not possible to receive any data transmitted across link $y$.

Here it is assumed that ACKs are not used. Rather, the modulation scheme is selected so that the successful data rate is maximized. The resulting data rate when links $a, b, .$. , and $c$ are also transmitting is denote by $R_{a, b, \ldots, c}^{N o A C K s}(x)$ and is given by

$$
\begin{aligned}
R_{a, b, \ldots, . c}^{N o A C K s}(x) & =\max _{m} \frac{Z \times 8}{\left(\frac{Z \times 8}{B R(m)}+F O H\right)} \\
& \times P S P_{Z}\left(m, S I N R_{a, b, \ldots, c}^{t, r}(x)\right)
\end{aligned}
$$

where $\mathrm{FOH}$ is fixed overhead that represents time spacings such as SIFS and radio synchronization.

\section{With Unsynchronized ACKs.}

If ACKs are used, the average number of transmissions until the data is successfully delivered across the link is $1 / P S P$. Thus, the effective data rate is approximately $B R \times P S P$ where it is assumed that exponential back-off is disabled. We consider two ways to ACK packets. In the first case, it is assumed that the ACK is transmitted just after the data packet is transmitted. This scheme is nearly the same as 802.11. One important difference between this case and 802.11 is that carrier sensing, RTS and CTS are not used. In this case, ACKs can be transmitted at any time; hence interference is due to both data and ACK transmissions. Thus, the SINR at the receiver of link $x$ is

$$
\begin{aligned}
& \operatorname{SINR}_{a, b, \ldots, c}^{t, r, U S A c k}(x)= \\
& \frac{H_{x, x}^{t, r}}{\max \left(H_{a, x}^{t, r}, H_{a, x}^{r, r}\right)+\ldots+\max \left(H_{c, x}^{t, r}, H_{c, x}^{r, r}\right)+N O I S E}
\end{aligned}
$$

where $H_{a, x}^{r, r}$ is the signal strength at the receiver of link $x$ due to an ACK transmission by the receiver of link $a$. Since a link cannot simultaneously transmit data and ACKs, $\max \left(H_{a, x}^{t, r}, H_{a, x}^{r, r}\right)$ is the worst case interference due to the data or ACK transmission across link $a$.

Similarly, the SINR experienced when receiving the ACK packets is

$$
\begin{aligned}
& \operatorname{SINR}_{a, b, \ldots, c}^{r, t, U S A c k}(x)= \\
& \frac{H_{x, x}^{r, t}}{\max \left(H_{a, x}^{t, t}, H_{a, x}^{r, t}\right)+\ldots+\max \left(H_{c, x}^{t, t}, H_{c, x}^{r, t}\right)+N O I S E}
\end{aligned}
$$

where $H_{a, x}^{t, t}$ and $H_{a, x}^{r, t}$ are the signal strengths at the transmitter of link $x$ due to the data transmission and the ACK transmission by link $a$ respectively.

Finally, if unsynchronized ACKs are used, links $a, b, \ldots$, and $c$ are transmitting, and the data packets are size $Z$, then the effective data rate across link $x$ is

$$
\begin{aligned}
& R_{a, b, \ldots, c}^{U S A c k}(x)=\max _{m, n} \frac{Z \times 8}{\left(\frac{Z \times 8}{B R(m)}+\frac{14 \times 8}{B R(n)}+2 F O H\right)} \\
& \times P S P_{Z}\left(m, S I N R_{a, b, \ldots c}^{t, r, U S A c k}(x)\right) \\
& \times P S P_{14}\left(n, S I N R_{a, b, \ldots c c}^{r, t, U S A c k}(x)\right)
\end{aligned}
$$

where it is assumed that ACKs have $14 \mathrm{~B}$, as is the case in 802.11. Closely related schemes to select the modulation fix the ACK modulation scheme to be the slowest bit-rate (i.e., $n=1$ ) or to require the data and ACK to sue the same bit-rate (i.e., $m=n$ ).

\section{With Synchronized ACKs.}

In the previous case, ACKs may interfere with data transmissions and vice versa. This interference can be eliminated 
if the ACK transmissions are synchronized. Specifically, we define

$$
\operatorname{SINR}_{a, b, \ldots, c}^{t, r, S A}(x)=\frac{H_{x, x}^{t, r}}{H_{a, x}^{t, r}+H_{b, x}^{t, r}+\ldots+H_{c, x}^{t, r}+N O I S E}
$$

and

$$
\operatorname{SINR}_{a, b, \ldots, c}^{r, t, S A}(x)=\frac{H_{x, x}^{r, t}}{H_{a, x}^{r, t}+H_{b, x}^{r, t}+\ldots+H_{c, x}^{r, t}+N O I S E} .
$$

Then the maximum data rate across link $x$

$$
\begin{aligned}
& R_{a, b, \ldots, c}^{S A}(x)=\max _{m, n} \frac{Z \times 8}{\left(\frac{Z \times 8}{B R(m)}+\frac{14 \times 8}{B R(n)}+2 F O H\right)} \\
& \times P S P_{Z}\left(m, S I N R_{a, b, \ldots c}^{t, r, S A}(x)\right) \\
& \times P S P_{14}\left(n, S I N R_{a, b, \ldots c}^{r, t, S A}(x)\right) .
\end{aligned}
$$

Note that while 802.11 uses ACKs, it is difficult to synchronize them in practice. Nonetheless, the model is included in our study in order to understand the impact of interference induced by ACKs.

\subsection{Protocol Communication Models}

In the above models, the data rate depends on the SINR, and the interference can be from multiple sources. One drawback of such models is that they do not easily lend themselves to representations as a graph. The protocol models are an alternative class of models that does allow graph-based analysis of communication and throughput algorithms. For this reason, the protocol model is widely used. The drawback of this model is that it does not accurately model interference.

Four types of protocol models are considered. In all cases, it is assumed that transmissions across a link can only occur if no transmission is occurring across any other links in a specific set of links. These links are referred to as the set of conflicting neighbors of the link. It is further assumed that a link can transmit at its full data rate if none of its conflicting neighbors are transmitting, where the full data rate is the achievable data rate if no link in the entire network is transmitting. On the other hand, if any link within its set of conflicting neighbors is transmitting, no transmission is possible. The difference between the various Protocol Communication Models is the set of conflicting neighbors and the full data rate.

\subsubsection{Node Exclusive Model}

The Node Exclusive Model is the simplest communication model. In this case, a link cannot transmit only if the transmitter or receiver is also involved in a transmission. Since $H_{x, y}^{t, r}=\infty$ implies that the transmitter of link $x$ is the receiver of link $y$, the set of conflicting neighbors in this case is

$$
\chi^{\text {NodeX }}(x):=\left\{\begin{array}{l}
y \mid \begin{array}{l}
H_{y}^{t, r}=\infty, H_{y, x}^{r, t}=\infty \\
H_{y, x}^{t, t}=\infty, H_{y, x}^{r, r}=\infty
\end{array}
\end{array}\right\} .
$$

It is assumed that if data transmissions are successfully received, then the receiver transmits an ACK, which must be correctly received in order to complete the data delivery. Thus, the (theoretical) effective data rate across link $x$ is

$$
\begin{aligned}
& R_{\varnothing}(x)=\max _{m, n} \frac{Z \times 8}{\left(\frac{Z \times 8}{B R(m)}+\frac{14 \times 8}{B R(n)}+2 F O H\right)} \\
& \times P S P_{Z}\left(m, S N R_{\varnothing}^{t, r}(x)\right) P S P_{14}\left(n, S N R_{\varnothing}^{r, t}(x)\right),
\end{aligned}
$$

where $S N R_{\varnothing}^{t, r}(x):=H_{x, x}^{t, r} / N O I S E$ and $S N R_{\varnothing}^{r, t}(x):=$ $H_{x, x}^{r, t} / N O I S E$, and we assume that the ACK is $14 \mathrm{~B}$. It is important to note that this data rate neglects interference and hence might not be achieved in practice.

Therefore, the data rate of link $x$ is

$$
R_{a, b, \ldots, c}^{\text {NodeX }}(x)=\left\{\begin{array}{l}
R_{\varnothing}(x) \text { if } a, b, . . c \notin \chi^{\text {NodeX }}(x) \\
0 \text { otherwise }
\end{array}\right.
$$

where $R_{\varnothing}(x)$ is given in (2).

\subsubsection{Two-Hop Node Exclusive Model}

The Node Exclusive Model ignores transmissions by nearby nodes. Consequently, this model greatly overestimates the physical layer's ability to withstand interference. This model can be made less optimistic by considering interference from transmissions that are "two hops" away either the transmitter or receiver. The definition of a hop is problematic. Specifically, even if the channel is quite poor and hence, the probability of successful transmission is near to, but greater than zero, if ACKs are used, then eventually a packet will be delivered, establishing a communication link. One approach is to define that nodes are one hop apart if the route forwards packet directly between the nodes. Thus, define $\mathcal{N}(\nu)$ to be the set of nodes that node $v$ transmits packet to or receives packets from. Define $v_{t}(x)$ and $v_{r}(x)$ to be the transmitter and receiver of link $x$, respectively. Then, the set of conflicting neighbors and the data rate of link $x$ are

$$
\begin{aligned}
& \chi^{2 H o p}(x):=\{y \mid \\
& \left.\left(N\left(\nu_{t}(x)\right) \cup N\left(\nu_{r}(x)\right)\right) \cap\left(N\left(\nu_{t}(y)\right) \cup N\left(\nu_{r}(y)\right)\right) \neq \varnothing\right\},
\end{aligned}
$$

and

$$
R_{a, b, \ldots, c}^{2 H o p}(x)=\left\{\begin{array}{l}
R_{\varnothing}(x) \text { if } a, b, \ldots, c \in \chi^{2 H o p}(x) \\
0 \text { otherwise }
\end{array}\right.
$$

\subsubsection{The Sensing Communication Model}

The sensing protocol model is perhaps the most widely examined communication model. In this case, a transmission across a link between nodes $\mathrm{A}$ and $\mathrm{B}$ cannot occur if there is some node $\mathrm{C}$ that is also transmitting where the received signal strength of C's transmission at either node A or B is above the Channel Sensing Threshold. This model is motivated by 802.11 . In 802.11 a node will only transmit if the channel is idle just before transmission. More specifically, the node will only transmit if the received signal strength of the aggregate of all other node's transmissions is below the Channel Sensing Threshold. Furthermore, since 802.11 uses either RTS-CTS-Data-ACK or Data-ACK, transmissions are two-way. Thus, in order for a transmission to occur, both the receiver and the transmitter must find the channel to be idle before transmissions.

The set of conflicting neighbors of link $x$ is denoted $\chi^{\text {Sense }}(x)$ and is given by

$$
\begin{aligned}
& \chi^{\text {Sense }}(x):= \\
& \left\{\begin{array}{l}
H_{y, x}^{t, r}>\text { Channel Sensing Threshold, } \\
H_{y, x}^{r, t}>\text { Channel Sensing Threshold, } \\
H_{y, x}^{t, t}>\text { Channel Sensing Threshold, or } \\
H_{y, x}^{r, x}>\text { Channel Sensing Threshold, }
\end{array}\right\} .
\end{aligned}
$$

This model can be interpreted in a slightly different way based on the Interference Range. In this model, a transmission across a link $x$ will fail if link $y$ is transmitting where $H_{y, x}^{t, r}>$ Interference Range. Since transmissions are bidirectional, this Interference Range model is the same as the 
Sensing Communication Model, but the Channel Sensing Threshold is replaced with the Interference Range.

The data rate of link $x$ is

$$
R_{a, b, \ldots, c}^{\text {Sense }}(x)=\left\{\begin{array}{l}
R_{\varnothing}(x) \text { if } a, b, \ldots, c \in \chi^{\text {Sense }}(x) \\
0 \text { otherwise }
\end{array}\right.
$$

\subsubsection{SINR Protocol Model}

The Sensing Protocol Model simplifies 802.11 by assuming that a transmission cannot occur from A to B if the received signal strength from any single node's transmission exceeds the Channel Sensing Threshold at either node A or B, and transmission will successfully occur at full rate otherwise. Thus, two simplifications are made.

1. The Sensing Protocol Model neglects the aggregate of the interference from multiple nodes transmitting.

2. The Sensing Protocol Model assumes that if the interfering signal strength is below particular Channel Sensing Threshold, then transmission at the full rate is possible.

This second simplification results from defining a single Channel Sensing Threshold for all links, where better performance would likely be achieved when such a parameter is determined on a per link basis. One alternative is to define that links are not in conflict if $S I N R$ at each receiver is above some threshold.

SINR protocol model is the protocol version of the physical model. Here, we provide a protocol version of the With Unsynchronized ACKs physical model in details. The Without ACKs and With Synchronized ACKs versions can be derived similarly.

\section{With Unsynchronized ACKs.}

Let $\mathcal{T}_{\text {data }}(x)$ and $\mathcal{T}_{A C K}(x)$ be the minimum allowable SINR the data transmissions and ACK transmissions. While there are many ways to set these thresholds, we set them via

$P S P_{Z}\left(m(x), \mathcal{T}_{\text {data }}(x)\right)=P S P_{Z}(m(x), S N R(x)) \times 0.99$

$P S P_{14}\left(n(x), \mathcal{T}_{A C K}(x)\right)=P S P_{14}(n(x), S N R(x)) \times 0.99$,

where $m(x)$ and $n(x)$ are the modulation schemes that solve (1), or the other schemes described just after (1) that restrict $m=n$ or $n=1$. These modulation selection schemes are named as OptDataAck, SameAck and MinAck, respectively.

The set of conflicting neighbors is

$$
\begin{aligned}
& \chi^{S I N R}(x):=
\end{aligned}
$$

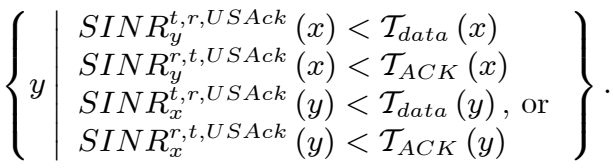

Note that Section 2.1.1 describes three ways to select the ACK rate (i.e., setting $n=1, n=m$ or optimizing over both $m$ and $n$ ). While selecting different ACK rates will not result in a significant change in the effective data rate, it will change the value of $\mathcal{T}_{A C K}(x)$, which may greatly impact $\chi^{S I N R}(x)$ and hence have a significant impact on spatial multiplexing.

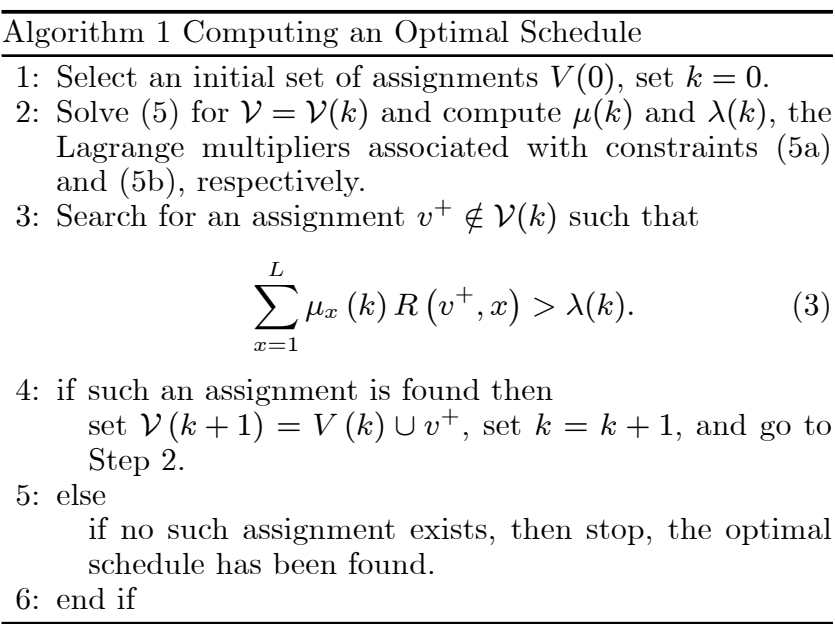

\section{THROUGHPUT OPTIMIZATION}

\subsection{System Model and Problem Formulation}

Let $\phi$ denote a particular connection, with $\Phi$ denoting the set of all such connections. The data rate along connection $\phi$ is denoted by $f_{\phi}$ and the path followed by connection $\phi$ is denoted by $P(\phi)$, that is, $P(\phi)$ is the set of links used by connection $\phi$, and the total data rate sent over link $x$ is $\sum_{\{(\phi) \mid x \in P(\phi)\}} f_{\phi}$, where $\{(\phi) \mid x \in P(\phi)\}$ is the set of flows that cross link $x$. All links are directional.

We define an assignment to be a vector $v=\left[\begin{array}{lll}v_{1} & \cdots & v_{L}\end{array}\right]$ where there are $L$ links in the network and where $v_{x} \in\{0,1\}$ with $v_{x}=1$ implying that link $x$ is transmitting during assignment $v$. The set of considered assignments is denoted by $\mathcal{V}$, while the set of all assignments is denoted $\overline{\mathcal{V}}$. Since $v_{x} \in\{0,1\}, \overline{\mathcal{V}}$ contains $2^{L}$ assignments. The size of $\dot{\overline{\mathcal{V}}}$ is the main challenging facing throughput maximization. Thus, typically, $\mathcal{V} \varsubsetneqq \overline{\mathcal{V}}$.

The data rate across link $x$ during assignment $v$ is denoted by $R(v, x)$. In general, $R(v, x)$ is a complicated function. However, here a simple binary relationship is used to define $R(v, x)$. Specifically,

$$
R(v, x)=\left\{\begin{array}{l}
R_{x} \text { if } v_{y}=0 \text { for all } y \in \chi(x) \\
0 \text { otherwise }
\end{array}\right.
$$

where $\chi(x)$ is a set of links that conflict with $x$, i.e., $y \in \chi(x)$ if simultaneous transmissions over $x$ and $y$ are not possible. $R_{x}$ is the nominal data rate over link $x$. Note that this definition of $R(v, x)$ neglects the possibility of transmission errors due to the aggregate interference from several links not in $\chi(x)$. The techniques to correct the aggregate interference is discussed in section 3.4.

The set of conflicting links, $\chi(x)$, depends on the communication model. It is assumed that all channel gains are constant. Since the focus of this paper is on the communication over the mesh infrastructure (which is not moving), such an assumption is reasonable.

A schedule is a convex combination of assignments. Specifically, a schedule is a set $\left\{\alpha_{v}: v \in \mathcal{V}\right\}$ where $\sum_{v \in \mathcal{V}} \alpha_{v} \leq 1$ and $\alpha_{v} \geq 0$. With this notation, the total data rate that the schedule $\alpha$ provides over link $x$ is $\sum_{v \in \mathcal{V}} \alpha_{v} R_{x} v_{x}$. Finally, 
the throughput optimization problem is

$$
\begin{aligned}
& \max _{\alpha, \mathbf{f}, F} F \\
& \text { subject to: } \\
& F \leq f_{\phi} \text { for all } \phi \in \Phi \\
& \sum_{\{\phi \mid x \in P(\phi)\}} f_{\phi} \leq \sum_{v \in \mathcal{V}} \alpha_{v} R(v, x) \text { for each link } x \\
& \sum_{v \in \mathcal{V}} \alpha_{v} \leq 1 \\
& 0 \leq \alpha_{v} \text { for each } v \in \mathcal{V},
\end{aligned}
$$

where $\mathbf{f}$ is the vector of flow rates.

As mentioned, the challenge in solving this problem is that optimality can be achieved if $\mathcal{V}=\overline{\mathcal{V}}$, but $\overline{\mathcal{V}}$ has $2^{L}$ elements. Alternatively, the set $\mathcal{V}$ can be constructed iteratively following Algorithm 1, which was presented in [2] to circumvent this problem of dimensionality. It can be proved that Algorithm 1 will converge to the optimal solution.

\subsection{Searching for New Assignments}

\subsubsection{MWIS}

The search for a new assignment required in Step 3 of Algorithm 1 can be accomplished by solving

$$
\max _{v} \sum_{x=1}^{L} R(v, x) \mu_{x},
$$

where $\mu_{x}$ is the Lagrange multiplier associated with constraint (5a). As will be shown next, solving this maximization is equivalent to finding the maximum weighted independent set of the weighted conflict graph.

A wireless network induces a conflict graph as follows. Each link in the network induces a vertex in the conflict graph. Thus, a link $x$ in the network is associated with a vertex in the conflict graph; this vertex is denoted with $x$, where whether $x$ refers to a link in the network or a vertex in the conflict graph is clear from the context. There is an edge between vertices $x$ and $y$ if $y \in \chi(x)$, where $x$ and links in $\chi(x)$ cannot simultaneously transmit. The weighted conflict graph is constructed by assigning the weight $R_{x} \mu_{x}$ to vertex $x$, where $R_{x}$ is the nominal data rate across link $x$.

An independent set (or stable set) of a graph is a set of vertices where no two vertices in the set are neighbors. Thus, an independent set of the conflict graph is a set of links that are not in conflict and hence, are able to transmit simultaneously. Letting $I$ be an independent set, the weight of $I$ is the sum of the weights of the vertices in $I$, i.e., $\sum_{x \in I} R_{x} \mu_{x}$. Since $I$ is an independent set, $\sum_{x \in I} R_{x} \mu_{x}=$ $\sum_{x=1}^{L} R(v(I), x) \mu_{x}$. Thus, solving (6) is equivalent to finding the maximum weighted independent set. The practical computational complexity of solving the MWIS problem is investigated in [8].

\subsubsection{Exact Algorithm for MWIS}

A useful way to compute the MWIS is to use Integer Linear Programming (ILP). Specifically, the MWIS problem can be written as

$$
\begin{aligned}
& \max _{\mathbf{v}} \sum R_{x} \mu_{x} \nu_{x} \\
& \text { such that: } v_{x}+\nu_{y} \leq 1 \text { if } y \in \mathcal{X}(x) \\
& v_{x} \in\{0,1\} .
\end{aligned}
$$

Note that since $v_{x} \in\{0,1\}$, this problem can also be solved with binary programming.

However, in large networks, there are many constraints (8). The computation time can be dramatically improved if clique decomposition is used. Specifically, a set of cliques $\left\{Q_{i}, i=1,2, \ldots M\right\}$ are found such that if $y \in \chi(x)$, then there is a clique $Q_{i}$ such that $x \in Q_{i}$ and $y \in Q_{i}$. Then, the MWIS problem becomes

$$
\begin{gathered}
\max _{\mathbf{v}} \sum_{x=1}^{L} R_{x} \mu_{x} v_{x} \\
\text { subject to: } \sum_{x \in Q_{i}} v_{x} \leq 1 \text { for } i=1,2, \ldots, M \\
v_{i} \in\{0,1\} .
\end{gathered}
$$

While an optimal clique decomposition might further improve the computation time, a simple greedy clique decomposition results in a factor of ten improvement over (7). There are many commercially available mixed integer and binary programming tools (e.g., CPLEX [5]). Our work has found that this method works well in practice, and is used mostly in our research.

\subsection{The Accuracy of Protocol Models}

While the protocol communication models have the significant benefit that the computational algorithms can be analyzed with graph theoretic means, these models suffer from drawback that they do not accurately represent interference. In order to gauge the impact of these approximations, the physical throughput of the schedules found from the protocol model were computed. That is, suppose that the optimal schedule based on the protocol model resulted in assignment $v$. In the protocol model, the data rates over link $x$ for this assignment is $R_{\varnothing}(x)$, where the $\varnothing$ denotes that the data rate is based on the assumption that no other node is transmitting.

When there are multiple active links in assignment $v$, it is unreasonable to ignore the interference because link $x$ may not achieve the normial data rate. Assume WithUnsyncACK physical model is used and the modulation schemes of the data and ACK of link $x$ are $(m, n)$, the actual link data rate is

$$
\begin{aligned}
& R_{\text {Actual }}\left(S I N R_{\text {data }}, S I N R_{\text {ack }}, m, n\right)= \\
& \frac{P S P_{Z}\left(m, S I N R_{\text {data }}\right) P S P_{14}\left(n, S I N R_{a c k}\right)}{\frac{1}{Z \times 8}\left(\frac{Z \times 8}{B R(m)}+\frac{14 \times 8}{B R(n)}+2 F O H\right)}
\end{aligned}
$$

where $S I N R_{\text {data }}, S I N R_{a c k}$ are the received $S I N R$ at the receiver and sender. With these actual link rates, the actual flow rate from the gateway(s) to each destination can be determined.

\subsection{Correcting Multi-Conflicts for SINR Protocol Model}

The protocol models may result in poor actual performance because they neglect interference from several sources and/or fail to fully exploit spatial multiplexing. However, due to the relationship between the protocol model and the MWIS problem, we seek to extend the protocol model to improve the actual performance. There are two approaches to correct the multi-conflicts problem. The first one is adding the multi-conflicts constraints during the optimal schedule searching process, and the second is to adjust the active link rate of the optimal schedule according to the actual $S I N R$. 


\subsubsection{Adding Multi-Conflicts Constraint}

An approach to eliminating multiple interfering links is to extend the Integer Linear Programming problem (9). Suppose that a newly found assignment specifies that links $y_{1}, \ldots, y_{k}$ and $x$ transmit simultaneously, but the accumulation of interference from $y_{1}, \ldots, y_{k}$ dramatically reduces the effective data rate across link $x$. In this case, the problem (9) is extended to

$$
\begin{aligned}
& \max _{\mathbf{v}} \sum_{x=1}^{L} R_{x} \mu_{x} v_{x} \\
\text { such that: } & \sum_{x \in Q_{i}} v_{x} \leq 1 \text { for } i=1,2, \ldots, M \\
& v_{x}+v_{y_{1}}+\ldots v_{y_{k}} \leq k \\
& v_{i} \in\{0,1\} .
\end{aligned}
$$

If further interferences are found, then further constraints can be added. To be convenient, this method is named as fixing multi-conflicts. Note that we still can adjust the link data rate for the optimal schedule even we correct the multiconflicts by adding multi-conflicts constraints.

\subsubsection{Adjust Active Link Rate for Optimal Schedul- ing}

Although adding multi-conflicts constraint can solve the multi-conflicts problem, it increases the number of solving MWIS problem and the corresponding computation time. It is possible to correct the multi-conflicts by adjusting the link data rate after we ignore the multi-conflicts and compute the optimal schedule.

Since the $S I N R_{\text {data }}, S I N R_{a c k}$ of each active link are known, we can adjust the link modulation schemes and eliminate the multi-conflicts. The modulation adjustment scheme $R_{\text {Opt }}\left(S I N R_{\text {data }}, S I N R_{a c k}\right)$ is defined as

$$
\begin{aligned}
& R_{O p t}\left(S I N R_{\text {data }}, S I N R_{a c k}\right)= \\
& \max _{m, n} R_{\text {Actual }}\left(S I N R_{\text {data }}, S I N R_{a c k}, m, n\right) .
\end{aligned}
$$

The flow rate can be determined from the new adjusted link rates.

\section{NUMERICAL EXPERIMENTS}

\subsection{Experimental Setup}

In order to investigate the performance of the different communication models, a large set of simulated urban mesh networks were generated. The radio propagation was determined from the UDelModels, a publicly available tool for computing urban propagation [1]. Ray-tracing was performed on the $2 \mathrm{~km}^{2}$ region of downtown Chicago, and the computation provided the received signal strength between any pair of nodes. Topologies were randomly generated by selecting a subset of nodes from a large baseline set of nodes. Nodes were placed to mimic a large infrastructure network. Specifically, outdoors, nodes were placed on lampposts throughout the city, and indoors, enough nodes were placed on each floor so that the entire floor was covered. In all, the baseline set of nodes included over 7000 nodes positioned throughout the city.

\section{Node Selection.}

Four parameters are used to construct a topology, namely, $n$ the number of nodes, $r^{*}$ the target bit-rates, $\Delta$ the maximum number of neighbors, and $N G W$ the number of gateways. The target bit-rate corresponds to specific received

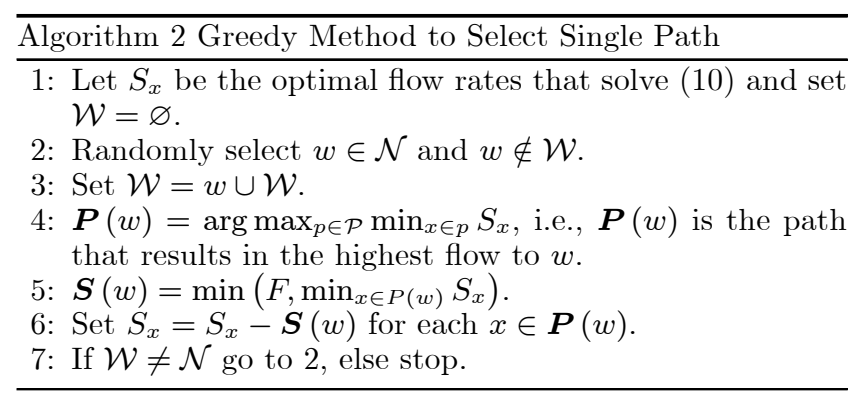

signal strength. Letting $R S S(r)$ be the minimum required received signal strength to decode a transmission at data rate $r$. We say that two nodes are neighbors if the propagation model results in a received signal strength that is above $R S S\left(r^{*}\right)$.

Let $N$ denote the set of nodes in the topology. Initially, $\mathcal{N}$ is a single node selected at random. Then, a node is selected at random among all the nodes that satisfy 1.) the node has between 1 and $\Delta$ neighbors in $\mathcal{N}$, and 2.) adding the node to $\mathcal{N}$ will not make any node in $\mathcal{N}$ have more than $\Delta$ neighbors in $\mathcal{N}$. If no such node exists, then the process is restarted. If suitable nodes do exist, the process continues until $\mathcal{N}$ has $n$ elements.

Next, gateways are selected. The objective is that the gateways are uniformly spread throughout the network in the sense that the average distance from a node to the closest gateway is minimized. A greedy algorithm developed was run ten times and the set of gateways that resulted in the smallest average distance was used.

\section{Routing.}

While there are several approaches to routing, this investigation uses a max-flow-based, interference aware routing. The first step in forming routes is to identify the set of potential links, their bit-rates, and the links that they interfere with. Let $x$ denote a link with transmitter $x_{t}$ and receiver $x_{r}$ and let $P_{x}$ be the received signal strength at the receiver. The bit-rate used by link $x$ is denoted $r(x)$ and is given by

$$
r(x):=\max \left\{r: P_{x}-P G \text { uard }>R S S(r)\right\},
$$

where PGuard is used as a buffer to reduce sensitivity to interference. This study used PGuard $=3 \mathrm{~dB}$. If no such bit-rate exists (i.e., $P_{x}-P G$ uard $<-90 \mathrm{dBm}$ ), then the link is removed from consideration.

Interference aware, multi-path max-flow routing is found by solving

$$
\begin{aligned}
& \max _{\mathbf{S}, F} F \\
& \sum_{\left\{x: x_{t}=w\right\}} S_{x}-\sum_{\left\{y: x_{r}=w\right\}} S_{y}+F=0 \text { for } w \notin G W \\
& \frac{S_{x}}{r(x)}+\sum_{y \in \chi(x)} \frac{S_{y}}{r(y)} \leq 1 \text { for all } x,
\end{aligned}
$$

where $S_{x}$ is the flow over link $x$. Note this optimization problem approximates the impact of interference. Specifically, $\frac{S_{x}}{r(x)}$ is the fraction of time that link $x$ transmits, and hence (10b) ensures that the fraction of time that link $x$ transmits and the fraction of times that all links that interfere with link $x$ transmit sum to no more than one. Of course, it is possible that some links that interfere with $x$ can transmit simultaneously. But (10b) does not account 


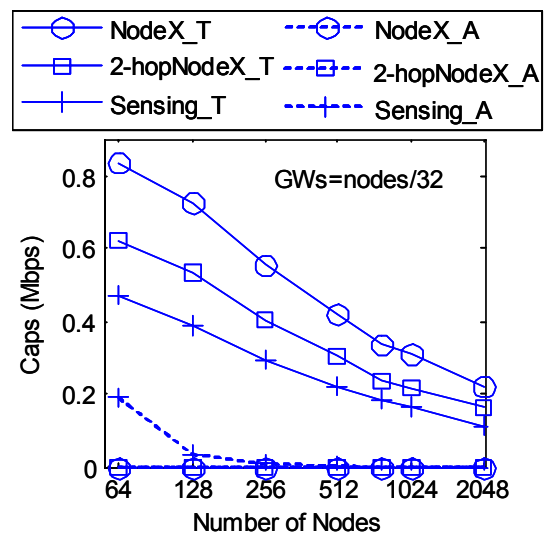

Figure 1: Computed theoretical and actual capacities for NodeX, 2-hop NodeX and Sensing models where the number of Gateways is equal to number of nodes divided by 32 .

for this possibility. Thus, (10) provides a lower bound on the throughput.

Problem (10) results in multi-path routing. Single path routing can be formed by quantization. Define $\mathcal{P}(w)$ to be the set of paths from some gateway to node $w$. Then the greedy algorithm shown in Algorithm 2 is used to construct $\boldsymbol{P}(w)$, a path from some gateway to node $w$.

\subsection{Performance of Communication Models}

Figure 1 shows the theoretical and actual throughput for Node Exclusive, 2-hop Node Exclusive, and Sensing models. The theoretical and actual capacities are the results from the optimal schedule that uses nominal link bit rate and actual link bit rate, respectively. The throughput is averaged over 40 samples for each topology, and the number of gateways is equal to the number of nodes divided by 32 . One complication with the Sensing model is that the Channel Sensing Threshold must be determined. In Figure 1, Channel Sensing Threshold $=-90 \mathrm{dBm}$. All of the models provide high theoretical capacities. However, since these models do not accurately represent the interference, it is not surprising that the actual throughputs are very poor. Specifically, Node Exclusive and 2-hop Node Exclusive models always have zero actual throughput, and Sensing model shows a small actual throughput when the network size is small and zero otherwise. The schedules from the Node Exclusive and the 2-hop Node Exclusive Models consistently result in no data traversing some links. Hence, the high theoretical throughput offered by the node exclusive model is fictitious.

Figure 2 shows the theoretical and actual capacities for SINR Protocol Model with Unsynchronized ACKs , Synchronized ACKs and Without ACKs, where the multi-conflicts are ignored and the MinAck selection scheme is used if ACK applied. As compared to use ACKS, not using ACKs eliminates overhead of ACK transmission, reduces the interference induced from ACK, and hence, achieves more spatial multiplexing. For these reasons, Without ACKs outperforms the schemes using ACKs. Note that this is the case even though the bit-rates selected with the Without ACKs scheme might be lower than schemes that use ACKs. The Synchronized ACKs case slightly outperforms the Unsynchronized ACKs because the synchronization eliminates the interference between data and ACK packets. The actual throughput of SINR Protocol Model is very close to the

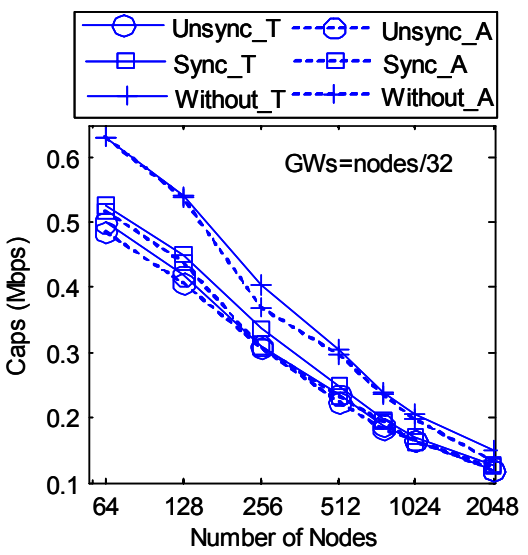

Figure 2: Computed theoretical and actual capacities for SINR Protocol Model with UnsyncAck, SyncAck and WithoutAck.

theoretical throughput. The reason for this behavior is that SINR Protocol Model accurately represents the interference.

\subsection{Performance of Correcting Multi-conflicts and Adjusting Bit-Rates}

There are two methods to correct the multi-conflicts for SINR protocol model. One way is to add multi-conflicts constraint, named as fixing multi-conflicts, during the process to find the optimal schedule. Another way is to ignore multiconflicts during the schedule optimization, but then adjust the link bit rates once the schedule and SINRs are known.

We consider three options to choose data and ACK transmission rates, namely MinAck, which uses the minimum ACK rate, SameAck, which uses the same data and ACK rate, and OptDataAck which might use the different data and ACK rates and selects the bit-rates to minimize the transmission time across a link (including retransmissions). These different methods correspond to the different ways to select the bit-rate for ACKs used in (1).

It should be emphasized that in this section we consider adjusting the bit-rates at two different stages. First, before the schedule optimization, the nominal data and ACK bitrates are selected as described in Section 2.2.4. Then, once the schedule has been computed, the bit-rates can be adjusted for each link and each assignment. In the first case, the bit-rates are selected based on SNR; while in the second case, the bit-rates are selected based on SINR. Also, in the first case, three possible ways of selected data and ACK bit-rates are considered, while in the second case, only the OptDataAck scheme is used. The reason for considering different schemes in the first case is that different schemes might result in different amounts of spatial multiplexing. However, once the schedule is determined, the spatial multiplexing is fixed.

Figure 3 shows the theoretical, actual, and adjusted throughputs for the three link modulation selection schemes where for each scheme multi-conflicts are either eliminated or not. The throughputs are normalized as follows. For each topology, the maximum actual throughput found over all six schemes but where the bit-rates are adjusted after the schedule has been determined. The right-hand frame shows the throughputs after the bit-rates have been adjusted. Thus, due to normalization, some of the throughputs are one. 


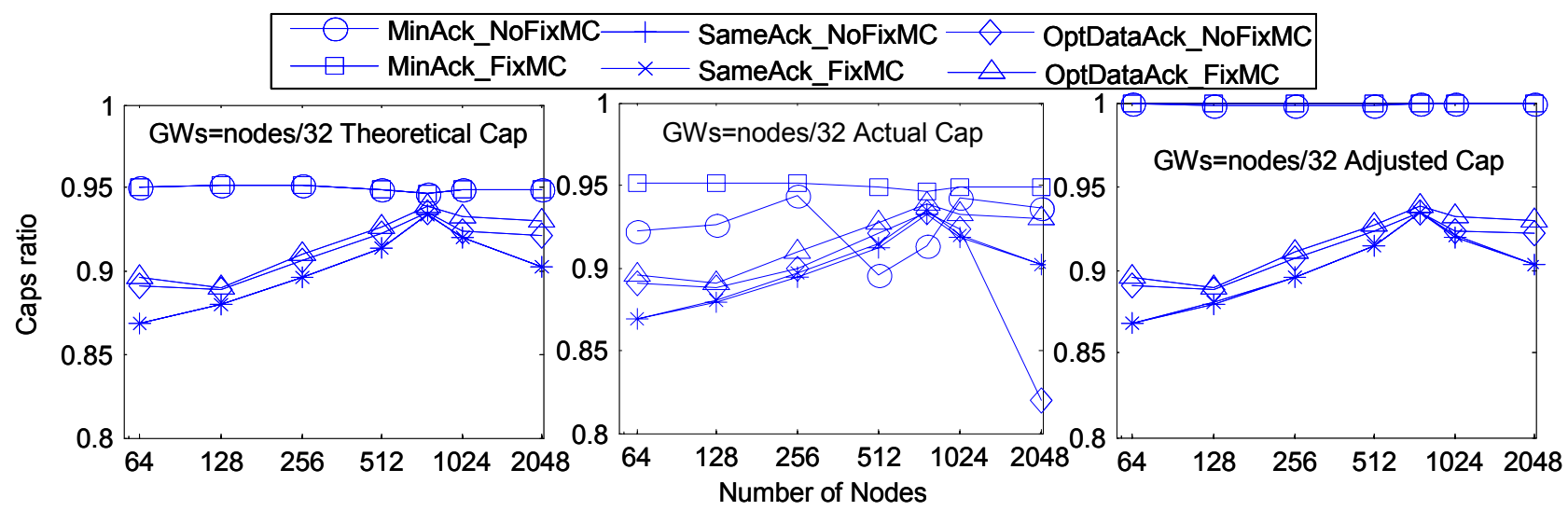

Figure 3: (a) The theoretical capacity for modulation selection schemes MinAck, SameAck and OptDataAck with fixing multi-conflicts or not (b) The actual capacity (c) The adjusted capacity

The left-hand frame in Figure 3 shows the theoretical throughput is not affected by whether multi-conflicts are fixed or not. On the other hand, the middle frame in Figure 3 shows that actual throughput of the schemes when multi-conflicts are ignored can be smaller than the theoretical throughput. For example, for the MinACK scheme, the actual throughput 3-5\% less than the theoretical, but for the OptDataACK scheme, the actual throughput is reduced by $12 \%$ when the topology has 2048 nodes. Also, as expected, the actual throughout is same as the theoretical throughout if we fix the multi-conflicts.

The right-hand frame of Figure 3 shows the throughput after the bit-rates are adjusted. In this case, there is minimal difference between accounting for multi-conflcits or not. Thus, in terms of throughput, if bit-rates are adjusted after the schedules are computed, multi-conflcits can be ignored. On the other hand, according to the algorithm described in Section 3.4, every time a multi-conflict is discovered, the MWIS problem must be changed and resolved. This can greatly increase the computational complexity. Thus, we conclude that multi-conflicts should be ignored.

Note that in the case of MinACK, the actual throughput after adjusting the bit-rates is about $5 \%$ larger than the theoretical throughput. This behavior is to due to suboptimal selection of the nominal bit-rates, which is corrected after the schedule is computed. This shows the utility of the simple procedure of adjusting bit-rates after schedules are computed.

Finally, note that the MinACK scheme achieves higher throughput than the other schemes. This behavior is expected since increasing the ACK rate does not greatly impact the effective data rate, but may significantly impact the set of conflicting links, and hence impact spatial multiplexing.

\section{CONCLUSIONS}

This paper explored communication models used in computing optimal throughput. It is found that in comparison to physical models, the protocol models enjoy the substantial computational advantage. However, the traditional protocol models, such as Node Exclusive, 2-hop Node Exclusive and Sensing protocol models have the drawback that they do not accurately model interference. Therefore, the actual throughput provided by these traditional protocol models is poor no matter how good the theoretical throughput offered.
A general SINR protocol model is proposed to more accurately represent the interference. Even if multi-conflicts are ignored, the SINR protocol model exhibits good throughput when applied to a physical model. If we employ techniques to correct multi-conflicts such as adding multi-conflicts constraints, then the final scheduling is feasible and the actual throughput is no worse than the theoretical one. Furthermore, if we adjust the link bit rate after the schedule is found, it is not necessary to fix the multi-conflicts by adding multi-conflicts constraints. This last approach provides the highest throughput along with fast computation.

\section{REFERENCES}

[1] Bohacek, S., Sridhara, V., and Kim, J. UDel Models. Available at: http://udelmodels.eecis.udel.edu/.

[2] Bohacek, S., and Wang, P. Toward tractable computation of the capacity of multihop wireless networks. In Infocom (2007).

[3] Chen, L., Low, S. H., and Doyle, J. C. Joint congestion control and media access control design for wireless ad hoc networks. In Proceedings of IEEE INFOCOM (March 2005), pp. $2212-2222$.

[4] Cruz, R., and Santhanam, A. Optimal routing, link scheduling and power control in multi-hop wireless networks. In IEEE INFOCOM (March 2003).

[5] ILOG. CPLEX. http://www.ilog.com/products/cplex/.

[6] Jain, K., Padhye, J., Padmanabhan, V., and Qiu, L. Impact of interference on multi-hop wireless network performance. In Proceedings of ACM MobiCom (San Diego, CA, September 2003), pp. 66-80.

[7] Lin, X., and Shroff, N. B. The impact of imperfect scheduling on cross-layer congestion control in wireless networks. IEEE/ACM Transactions on Networking 14, 2 (April 2006), 302-315.

[8] Wang, P., and Bohacek, S. On the practical complexity of solving the maximum weighted independent set problem for optimal scheduling in wireless networks. In WICON submitted (2008). 